\title{
Inhibitors of antibiotic resistance mechanisms: clinical applications and future perspectives
}

\author{
Barbara Parrino ${ }^{1}$, Daniela Carbone ${ }^{1}$, Girolamo Cirrincione $^{1}$, Patrizia Diana ${ }^{1}$ \& Stella \\ Cascioferro*, 1 \\ ${ }^{1}$ Dipartimento di Scienze e Tecnologie Biologiche Chimiche e Farmaceutiche (STEBICEF), Università degli Studi di Palermo, Via \\ Archirafi 32, 90123 Palermo, Italy \\ *Author for correspondence: stellamaria.cascioferro@unipa.it
}

"Antivirulence strategies can be considered a promising approach to overcome the global threat of antibiotic resistance as this allows you to deprive the bacterium of its pathogenicity without interfering with its life cycle."

First draft submitted: 15 November 2019; Accepted for publication: 5 December 2019; Published online: 6 February 2020

Keywords: anti-biofilm agents $\bullet$ antibiotic resistance $\bullet$ antivirulence strategy $\bullet$ ESKAPE pathogens

Bacterial strains responsible for antibiotic resistant infections are increasing in an alarming way and the evolution of resistance mechanisms seems to be unstoppable. In the past decade, many efforts have been made in order to counteract this phenomenon but very few compounds have reached clinical trials. The development of new classes of antibiotics able to overcome the main bacterial drug resistance mechanisms is urgently required to counter the imminent danger of a postantibiotic era [1].

Scientific research should be oriented toward the discovery of new antimicrobial agents with specific features, including the capability to kill pathogens selectively without affecting the microbiota and to interfere with important virulence factors such as the biofilms [2]. Therapeutic strategies targeting virulence factors without interfering with microbial growth were recognized as a valuable alternative to conventional antibiotics as they should impose lower selectivity pressure on the rise of antibiotic-resistance strains [3]. Despite the many advances that have been made in this field, the lack of in vivo experiments to assess the clinical potential of the new antivirulence agents still remains one of the main drawbacks.

Serious chronic infections, especially in hospital settings, are frequently caused by six bacterial pathogens known with the acronym of their names, ESKAPE (Enterococcus faecium, Staphylococcus aureus, Klebsiella pneumoniae, Acinetobacterbaumannii, Pseudomonas aeruginosa and Enterobacter spp.). Understanding the mechanisms of antimicrobial resistance in these pathogens is crucial for identifying new strategies to treat drug-resistant infections.

The biochemical mechanisms of antibiotic resistance such as enzymatic resistance, which causes the inactivation of the antibiotics and efflux pumps responsible for the extrusion of the drug from the bacterial cell, were considered valuable drug targets for add-on treatment of bacterial infections.

The expression of the efflux pumps could be considered one of the main mechanisms of resistance against antibiotics in both Gram-positive and Gram-negative pathogens.

In the past decade, many efforts have been made to develop new potent efflux pump inhibitors (EPIs). A wide number of chemical compounds belonging to various chemical families, including quinolines, quinolones, naphtene compounds, boronic acid derivatives, chalcones and indole derivatives, have been described for their significant efflux pump inhibition activity. Representative examples of synthetic small molecules with EPI activity are the naphtene derivatives, described by Thota $e t$ al. as 3-(3,4-dihydronaphthalen-2-yl)-propenoic acid isobutyl and N,N-diisopropylamide amides, which act as NorA inhibitors reducing the minimum inhibitory concentration (MIC) value of ciprofloxacin (CPX) from 8 to $0.5 \mu \mathrm{g} / \mathrm{ml}$ against NorA overexpressing S. aureus 1199B [4]. Among boronic acids, Fontaine et al. identified 6-benzyloxypyridine-3-boronic acid and 4-benzyloxyphenyl boronic acid as 
new promising NorA inhibitors, which showed a significant synergistic activity with CPX against S. aureus $1199 \mathrm{~B}$ with $\mathrm{MMC}_{4}$ (minimum modulatory concentration of inhibitor to achieve a fourfold CPX MIC reduction) values of 1 and $0.5 \mu \mathrm{g} / \mathrm{ml}$, respectively [5].

Even though the combination of EPIs and antibiotics was associated with an improvement of the antibacterial activity and the antimicrobial spectrum of antibiotics, no EPIs reached the clinic, despite a promising topical use of such inhibitors. This is due to their high toxicity and low in vivo efficacy [6].

Moreover, an ideal candidate as a new antimicrobial agent should be active against the planktonic form of life, as well as active against the biofilm phenotype. Biofilms are currently considered one of the most important bacterial virulence factors, which are responsible for serious nosocomial infections resistant to conventional antibiotics [7]. Structurally, biofilms are microbial communities in which the bacterial cells are enclosed into a matrix constituted of extracellular polymeric substance such as polysaccharides, proteins, lipids and extracellular DNA (e-DNA) [8].

Despite the growing knowledge of the biofilm organization and the important results obtained in the past decade in this research field, the treatment of biofilm-associated infections remains an important challenge. Recently, many compounds endowed with potent in vitro antibiofilm activity were described [9-11], but, unfortunately, no derivative is close to clinical trials. This is generally due to the lack of in vivo experiments aimed to confirm their antivirulence properties and their protective effects against infections caused by bacterial-resistant strains.

Three main mechanisms of action are identified as responsible for antibiofilm activity: the inhibition of the bacterial adhesion to surfaces, which is the first step of bacterial pathogenesis, the disruption of the biofilm architecture during its maturation process and the interference with the quorum sensing system [12,13].

The most relevant targets involved in the antibiofilm activity are: quorum sensing signalling molecules such as $N$-acylhomoserine lactone in Gram-negative pathogens, the autoinducing peptides described in Gram-positive bacteria and autoinducer- 2 which was found in both Gram-negative and Gram-positive bacteria; nucleotide second messenger signaling systems including cyclic dimeric guanosine monophosphate and the cyclic dimeric adenosine monophosphate [14]; a modified nucleotide, tetra and pentaphosphate guanosine derivative of GTP and GDP known as (p)ppGpp and finally, targets concerning the microbial attachment to the surfaces such as the transpeptidase Sortase A for Gram-positive pathogens and type 1 fimbriae for Gram-negative strains [2].

Many classes of compounds were described in the past decade for their interesting antibiofilm properties, including imidazole compounds, indoles, carbazoles, phenazine and quinoline derivatives. Of these, the imidazole scaffold was the most investigated. The most significant contribution was given by Melander and coworkers who synthesized several libraries of 2-aminoimidazole derivatives that are able to prevent biofilm formation or disperse mature biofilms at low micromolar concentrations through mechanisms which do not affect microbial growth, therefore showing a typical antivirulence profile [15].

Another relevant class of heterocyclic compounds was represented by indole derivatives, which are widely described for their numerous biological activities, in particular, for the anticancer [16,17] and antibacterial [11] properties. Indole 5-haloaldonitrones were described as NorA inhibitors with synergistic effect with CPX against SA$1199 \mathrm{~B}$ showing MIC values in the range $0.5-2 \mu \mathrm{g} / \mathrm{ml}$ in combination with subinhibitory (MIC/4) concentrations of the antibiotic [18].

Despite important progress that has been made to counteract the antibiotic resistance, including the understanding of the main molecular mechanisms at the base of the onset of resistant bacterial strains, we are still very far from identifying an effective and safe strategy that can reach the clinical phase. Different aspects must be taken into consideration to identify reliable candidates to fight multi-drug-resistant (MDR) strains. In the case of new compounds interfering with the microbial viability, the studies on the spontaneous frequencies of resistance were particularly relevant as they allow for the establishment of the capability of the compounds to promote antibiotic resistance and their suitability to be developed into new antibacterial drug.

Antivirulence strategies can be considered a promising approach to overcome the global threat of antibiotic resistance as this allows you to deprive the bacterium of its pathogenicity without interfering with its life cycle. Among the virulence factors, bacterial biofilms certainly represent one of the most valuable targets. In vivo studies aiming to validate new antibiofilm drugs are urgently needed for counteracting the emergence of chronic biofilmassociated infections. 


\section{Financial \& competing interests disclosure}

The authors have no relevant affiliations or financial involvement with any organization or entity with a financial interest in or financial conflict with the subject matter or materials discussed in the manuscript. This includes employment, consultancies, honoraria, stock ownership or options, expert testimony, grants or patents received or pending, or royalties.

No writing assistance was utilized in the production of this manuscript.

\section{References}

1. Schillaci D, Spanò V, Parrino B et al. Pharmaceutical approaches to target antibiotic resistance mechanisms. J. Med. Chem. 60(20), 8268-8297 (2017).

2. Parrino B, Schillaci D, Carnevale I et al. Synthetic small molecules as anti-biofilm agents in the struggle against antibiotic resistance. Eur. J. Med. Chem. 161, 154-178 (2019).

3. Parrino B, Diana P, Cirrincione G, Cascioferro S. Bacterial biofilm inhibition in the development of effective anti-virulence strategy. Open Med. Chem. J. 12(1), 84-87 (2018).

4. Thota N, Reddy MV, Kumar A et al. Substituted dihydronaphthalenes as efflux pump inhibitors of Staphylococcus aureus. Eur. J. Med. Chem. 45(9), 3607-3616 (2010).

5. Fontaine F, Héquet A, Voisin-Chiret A-S et al. Boronic species as promising inhibitors of the Staphylococcus aureus NorA efflux pump: study of 6-substituted pyridine-3-boronic acid derivatives. Eur. J. Med. Chem. 95, 185-198 (2015).

6. Wang Y, Venter H, Ma S. Efflux pump inhibitors: a novel approach to combat efflux-mediated drug resistance in bacteria. Curr. Drug Targets 17(6), 702-719 (2016).

7. del Pozo JL, Patel R. The Challenge of treating biofilm-associated bacterial infections. Clin. Pharm. Ther. 82(2), 204-209 (2007).

8. Kim S-K, Lee J-H. Biofilm dispersion in Pseudomonas aeruginosa. J. Microbiol. 54(2), 71-85 (2016).

9. Cascioferro S, Parrino B, Petri GL et al. 2,6-Disubstituted imidazo[2,1-b][1,3,4] thiadiazole derivatives as potent staphylococcal biofilm inhibitors. Eur. J. Med. Chem. 167, 200-210 (2019).

10. Roy R, Tiwari M, Donelli G, Tiwari V. Strategies for combating bacterial biofilms: a focus on anti-biofilm agents and their mechanisms of action. Virulence 9(1), 522-554 (2018).

11. Carbone A, Parrino B, Cusimano MG et al. New thiazole nortopsentin analogues inhibit bacterial biofilm formation. Mar. Drugs 16(8), 274 (2018).

12. Chung PY, Toh YS. Anti-biofilm agents: recent breakthrough against multi-drug resistant Staphylococcus aureus. Pathog. Dis. 70(3), 231-239 (2014).

13. Cascioferro S, Cusimano MG, Schillaci D. Antiadhesion agents against Gram-positive pathogens. Future Microbiol. 9, 1209-1220 (2014).

14. Corrigan RM, Abbott JC, Burhenne H, Kaever V, Gründling A. c-di-AMP is a new second messenger in Staphylococcus aureus with a role in controlling cell size and envelope stress. PLoS Pathog. 7(9), e1002217 (2011).

15. Furlani RE, Yeagley AA, Melander C. A flexible approach to 1,4-di-substituted 2-aminoimidazoles that inhibit and disperse biofilms and potentiate the effects of $\beta$-lactams against multi-drug resistant bacteria. Eur. J. Med. Chem. 62(Suppl. C), 59-70 (2013).

16. Parrino B, Carbone A, Spanò V et al. Aza-isoindolo and isoindolo-azaquinoxaline derivatives with antiproliferative activity. Eur. J. Med. Chem. 94, 367-377 (2015).

17. Parrino B, Carbone A, Ciancimino $\mathrm{C}$ et al. Water-soluble isoindolo[2,1-a]quinoxalin-6-imines: in vitro antiproliferative activity and molecular mechanism(s) of action. Eur. J. Med. Chem. 94, 149-162 (2015).

18. Burchak ON, Pihive EL, Maigre L et al. Synthesis and evaluation of 1-(1H-indol-3-yl)ethanamine derivatives as new antibacterial agents. Bioorg. Med. Chem. 19(10), 3204-3215 (2011). 
